\title{
Study of electrical behavior of liquid crystal devices doped with titanium dioxide nanoparticles
}

\author{
V. Marzal, ${ }^{1}$ J. C. Torres, ${ }^{* 1}$ B. García-Cámara, ${ }^{1}$ I. Pérez, ${ }^{1}$ J. M. Sánchez-Pena, ${ }^{1}$ and W. Piecek ${ }^{2}$ \\ ${ }^{1}$ Group of Displays and Photonic Applications. Carlos III University of Madrid, 28911, Leganés, Spain \\ ${ }^{2}$ Institute of Applied Physics, Military University of Technology, Warsaw, Poland.
}

Received February 15, 2017; accepted February 22, 2017; published March 31, 2017

\begin{abstract}
In the last years, nanostructures are widely used as dopants in liquid crystals to manipulate either their electrical or optical properties. In this work, we have analyzed the electrical response of a planar cell filled with a mixture of E7 liquid crystal doped with $\mathrm{TiO}_{2}$ nanoparticles. The effect of these dopants on the effective permittivity and conductivity of the cell has been studied in a wide frequency range at different temperatures.
\end{abstract}

Nowadays, devices based on liquid crystal (LC) materials are widely used. Liquid crystal displays (LCDs) dominate the display market and other applications of LC devices (optical filters and switches, microwave and security devices) [1-3] have been developed in the last decades.

In order to improve the electro-optical behavior of these devices, considerable attention has been paid recently to analyze the influence of incorporating nanoscale materials on an LC host. The modification of the behavior of the LC depends on the nanoparticles (NPs) used as guest dopants. Many research studies on the electro-optical response of LC-NPs mixtures using metallic [4-5], insulating [6-7] or ferroelectric [8] nanoparticles or carbon nanotubes (CNTs) [9] can be found in the literature. These works reveal that LC-NPs mixtures can exhibit enhancement of some characteristics like dielectric anisotropy, birefringence and threshold voltage, among others. In addition, LC has been also shown as an interesting active medium to govern the optical response of guest NPs, e.g. actively shifting the spectral position of scattering resonances [10].

In this work, a study of the frequency-dependent electrical properties of liquid crystal E7 doped with $\mathrm{TiO}_{2}$ nanoparticles is presented. The temperature dependence of these properties is also analyzed, including the behavior of the mixture when the LC material E7 is in the isotropic phase. Experimental results show that doping the LC material E7 with $\mathrm{TiO}_{2}$ nanoparticles substantially modifies its dielectric properties.

A composite of E7 LC doped with $\mathrm{TiO}_{2}$ nanoparticles of 250nm (C) Sigma-Aldrich, concentration 53-57wt.\% in diethylene glycol monobutyl ether/ethylene glycol) was

*jctzafra@ing.uc3m.es obtained using the following procedure. Nanoparticles were mixed in pure liquid crystal with a 1:2 proportion, although only a small part of $\mathrm{TiO}_{2}$ NPs are finally mixed. After that, the mixture was stirred for $1 \mathrm{~min}$ till the solution was homogeneously mixed. Thereafter, the solution was left for 6 hours at a temperature of $80^{\circ} \mathrm{C}$ in order to evaporate completely the organic solvent.

LC cells were filled with the final mixture by capillary action, with the LC in the isotropic phase. To obtain a uniform distribution of the mixture inside the cell, the sample was cooled slowly. LC cells were composed of two glass plates, $5 \mu \mathrm{m}$ apart. Both plates were covered with transparent conductive films of indium tin oxide (ITO) as electrodes. A thin layer of polyimide SE-130 with parallel rubbing was used as an alignment layer for the LC in both substrates.

Experimental measurements were performed using the setup displayed in Fig. 1. The magnitude and phase of the impedance of planar LC cells were measured using a Solartron 1260 impedance analyzer. A TS102 programmable thermal stage with an mK2000 temperature controller is used to keep the temperature of samples constant. Measurements were made at several frequencies in the range from $20 \mathrm{~Hz}$ to $1 \mathrm{MHz}$, using a sinusoidal voltage signal with $100 \mathrm{mV}_{\text {RMS }}$. A set of temperatures $\left(30^{\circ} \mathrm{C}, 50^{\circ} \mathrm{C}\right.$ and $\left.70^{\circ} \mathrm{C}\right)$ was checked.

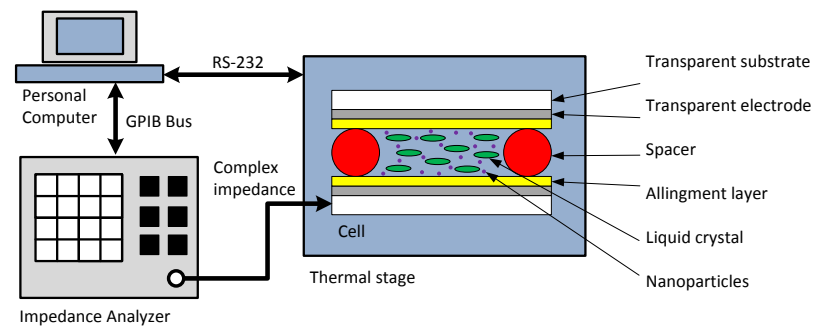

Fig. 1. Experimental setup to acquire impedance measurements.

From an electrical point of view, the LC cell can be modeled as a parallel-plate capacitor with a non-ideal dielectric, i.e. the $\mathrm{TiO}_{2}$-doped $\mathrm{E} 7 \mathrm{LC}$ mixture, and therefore the electrical response of the LC sample can be 
approximated as equivalent to that of a capacitor with a loss resistor in parallel [11]. The admittance, Y, of this equivalent circuit can be expressed as a function of the measured impedance magnitude, $|\mathrm{Z}|$, and phase, $\varphi$, as follows:

$$
Y=\mathrm{j} \omega C_{0}\left(\varepsilon_{\perp}{ }^{\prime}-\mathrm{j} \varepsilon_{\perp}{ }^{\prime \prime}\right)=\frac{1}{|Z|} \cos (\varphi)-\mathrm{j} \frac{1}{|Z|} \sin (\varphi),
$$

where $\omega$ is the frequency of the applied sinusoidal voltage, $C_{0}$ is the vacuum device capacitance, and $\varepsilon_{\perp}{ }^{\prime}$ and $\varepsilon_{\perp}$ " are, respectively, the real and imaginary parts of the complex dielectric constant of LC planar cells. Then, $\varepsilon_{\perp}$ ' and $\varepsilon_{\perp}$ "' can be deduced from impedance measurements as:

$$
\begin{aligned}
& \varepsilon_{\perp}{ }^{\prime}=-\frac{\sin \varphi}{|Z| \cdot \omega \cdot C_{0}} \\
& \varepsilon_{\perp}{ }^{\prime \prime}=\frac{\cos \varphi}{|Z| \cdot \omega \cdot C_{0}}
\end{aligned}
$$

and the imaginary part of permittivity was used to

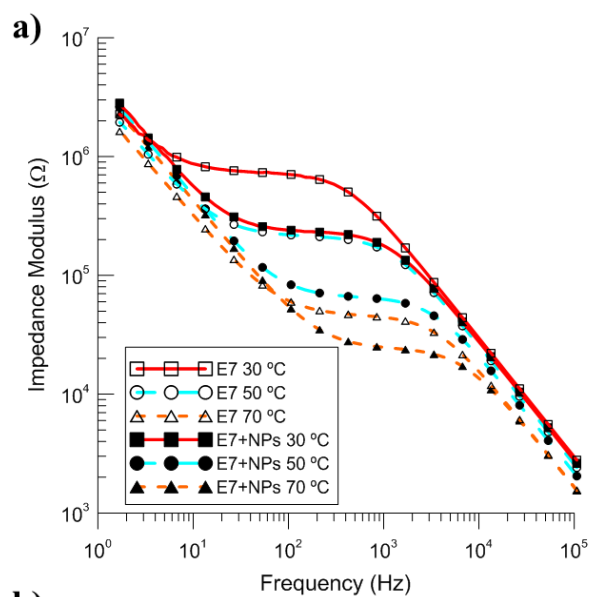

b)

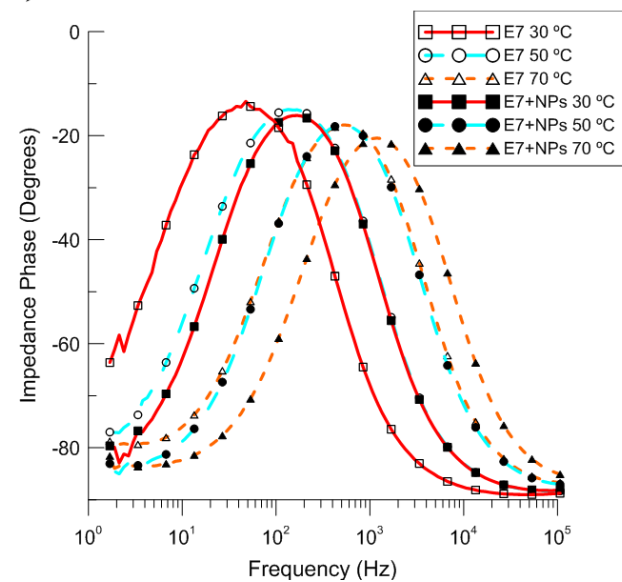

Fig. 2. Frequency dependence of impedance, both modulus (a) and phase (b) of cell filled with pure E7 LC samples (hollow symbols) or with a $\mathrm{TiO}_{2}$ - doped mixture (solid symbols). Measurements also consider different temperatures. calculate the effective conductivity $(\sigma)$ of the cell, using the following equation:

$$
\sigma=\omega \varepsilon_{\mathrm{o}} \varepsilon
$$

where $\varepsilon_{\mathrm{o}}$ is the vacuum permittivity. The impedance, both modulus (Fig. 2a) and phase (Fig. 2b), of cells with pure E7 $\mathrm{LC}$ or $\mathrm{TiO}_{2}$-doped mixture are plotted in Fig. 2 as a function of the frequency for different temperatures.

The influence of the nanoparticles can be strongly noticed in the electrical response of the LC cell, as it can be seen in $[12,13]$. In particular, Fig. 3 shows the effective permittivity, both for real (Fig. 3a) and imaginary (Fig. $3 \mathrm{~b})$ parts of the mixture and the conductivity of the cell (Fig. 3c) as a function of the frequency and for different temperatures. Temperatures, such as when E7 is either in the nematic $\left(30^{\circ} \mathrm{C}\right.$ and $\left.50^{\circ} \mathrm{C}\right)$ or the isotropic phase $\left(70^{\circ} \mathrm{C}\right)$, have been considered. In addition, the corresponding results of an undoped E7 LC cell have been shown for comparison.

The presence of $\mathrm{TiO}_{2}$ nanoparticles in the composite clearly shows a global increment of the real part of permittivity, but there is also an effect on frequency response. It is known that pure liquid crystal samples have a larger value of $\varepsilon$ ' at low frequencies, which decreases as frequency increases until a plateau region at high frequencies (Fig. 3a). This is because at low frequencies electric permittivity mainly depends on orientational polarization contribution and space charge polarization, which increases with decreasing frequencies [12]. In our samples, the addition of $\mathrm{TiO}_{2}$ nanoparticles reveals reinforcement of the inherent dipolar moment of LC molecules through the induced dipole moment of the NP, producing an increment of dielectric permittivity. This is also seen in the imaginary part, $\varepsilon_{\perp}$ " (Fig. 3b).

The presence of NP also influences the frequency response of $\varepsilon_{\perp}$ '. The transition frequency of $\varepsilon_{\perp}$ ' is shifted at larger frequencies (Fig. 3a), while the peak in $\varepsilon_{\perp}$ ", related to a Debye relaxation process is also moved to high frequencies when there are $\mathrm{TiO}_{2}$ NPs in the mixture. Regarding the temperature dependence, it can be seen that in a pure E7 sample in the nematic phase, the frequency response of the cell is shifted to higher frequencies as temperature increases, while the value of the real part of dielectric permittivity slightly varies in the plateau region. This may be attributed to a shorter relaxation time and increasing charges confirmed by the conductivity data (Fig. 3c). In samples with NPs, this frequency shift is enhanced. However, it is slightly smaller in the isotropic phase $\left(70^{\circ} \mathrm{C}\right)$, than in the nematic one $\left(30^{\circ} \mathrm{C}, 50^{\circ} \mathrm{C}\right)$. 


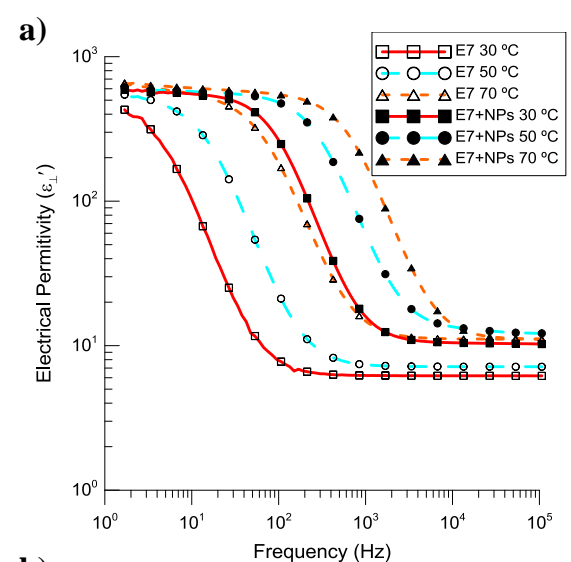

b)
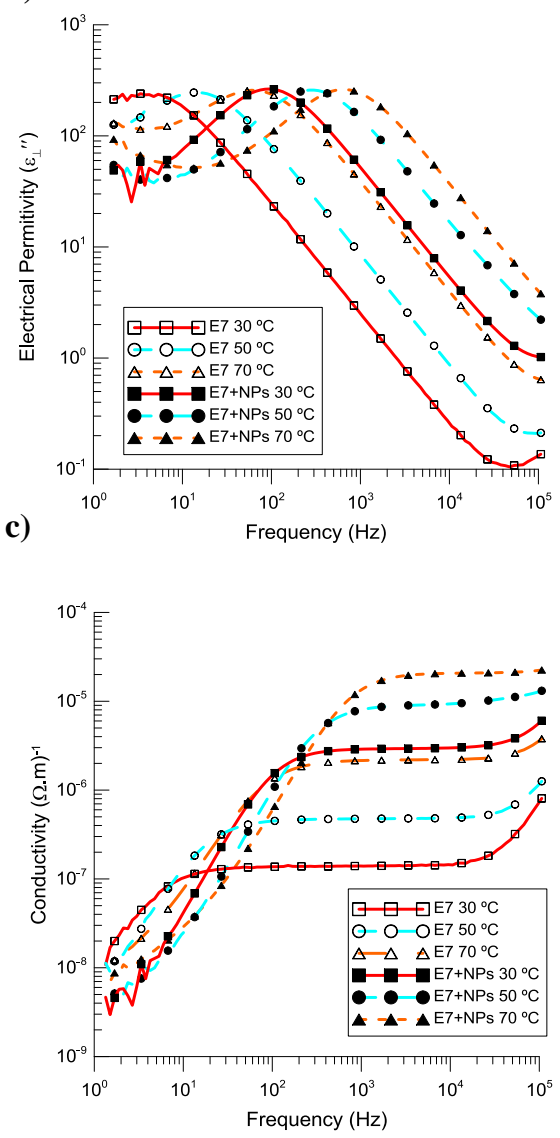

Fig. 3. Experimental data of the a) real part of dielectric permittivity, b) imaginary part of dielectric permittivity, and c) conductivity of $\mathrm{E} 7$ and the $\mathrm{E} 7+\mathrm{TiO}_{2}$ nanoparticles cells as a

As expected, the variation of conductivity (Fig. 3c) increases with temperature in pure E7 samples as the mobility and number of free ions increase with temperature. In doped samples, this increment is remarkably larger showing that the $\mathrm{TiO}_{2}$ concentration is low enough to produce a free-ion-trapping phenomenon
[13]. On the contrary, its presence reinforces the number of free ions increasing the global conductivity.

In summary, we showed the frequency response of effective permittivity, real and imaginary parts, and the conductivity of composite of E7 LC doped with $250 \mathrm{~nm}$ $\mathrm{TiO}_{2}$ nanoparticles. The considered samples showed a global increment of the effective real part of the permittivity of the mixture, while it is also globally shifted to higher frequencies. On the other hand, we showed that increment of the conductivity of the sample as adding $\mathrm{TiO}_{2}$ NPs, concluding that dopants are reinforcing the presence and mobility of free ions, instead of trapping them. In addition, this behavior is observed at different temperatures, either in the nematic or the isotropic phase.

The authors want to acknowledge the support by Ministerio de Economia y Competitividad of Spain (TEC2013-47342-C2-2-R and TEC2016-77242-C3-1-R) the RD Program of the Comunidad de Madrid (SINFOTON S2013/MIT-2790) and COST Action IC1208.

\section{References}

[1] P.J. Pinzón, I. Pérez, C. Vázquez, J.M.S. Pena, App.Opt. 51, 5960 (2012)

[2] C. Carrasco-Vela, X. Quintana, E.Otón, M.A. Geday, J.M. Otón, Opto-Electron. Review 19, 496 (2011).

[3] J. Torrecilla, E. Ávila-Navarro, C. Marcos, V. Urruchi, J.M.S. Pena, J. Arias, M.M Sánchez-López, Microw. Opt. Technol. Lett. 55, 2420 (2013).

[4] G.B. Hadjichristov, Y.G. Marinov, A.G. Petrov, E. Bruno, L.Marino, N. Scaramuzzab, Mol. Cryst. Liq. Cryst. 610, 135 (2015).

[5] T. Miyama, J. Thisayukta, H. Shiraki, Y. Sakai, Y. Shiraishi, N. Toshima, S. Kobayashi, Jpn. J. Appl. Phys. 43, 2580 (2004).

[6] W.T. Chen, P.S. Chen, C.Y. Chao, Jpn. J. Appl. Phys. 48, 015006 (2009).

[7] A. Siarkowska, M. Chychłowski, T.R. Woliński, A.Dybko. Phot. Lett. Poland 8, 29 (2016).

[8] O. Buchnev, A. Dyadyusha, M. Kaczmarek, V.Reshetnyak, Y. Reznikov, J. Opt. Soc. Am. 24, 1512 (2004).

[9] A. García-García, R. Vergaz, J.A. Algorri, X. Quintana, J.M. Otón, Beilstein J. Nanotechnol. 6, 396 (2015).

[10] R. Pratibha, K. Park, I.I. Smalyukh, W. Park, Opt. Express 17,19459 (2009).

[11] J.C. Torres, B. Garcia-Camara, I. Perez, V. Urruchi, J.M. Sanchez-Pena, Sensors 15, 5594 (2015).

[12] P. Kumar, A. Kishore and A, Sinha, Adv. Mater. Lett. 7, 104 (2016).

[13] R.K. Shukla, C.M. Liebig, D.R. Evans, W. Haase, RSC Adv. 4, 18529 (2014). 